\title{
Die subalpine Gebirgsflora in Teneriffas Nationalpark Teide
}

\author{
Rolf Goetz
}

\begin{abstract}
The Teide National Park is located in the center of the Canary Island of Tenerife. With up to 4 million visitors per year, it is the most visited tourist attraction of the Canary Islands. In 2007, the region was declared a World Heritage Site by UNESCO. In addition to a spectacular volcanic landscape, the protected area is characterized by the extraordinary mountain flora with numerous endemic plants. The most important species are briefly presented.
\end{abstract}

\section{Zusammenfassung}

Der Teide-Nationalpark befindet sich im Zentrum der Kanareninsel Teneriffa. Mit jährlich bis zu 4 Millionen Besuchern ist er die am meisten besuchte Touristenattraktion der Kanarischen Inseln. 2007 wurde die Region von der UNESCO zum Weltnaturerbe erklärt. Neben einer spektakulären Vulkanlandschaft zeichnet sich das Schutzgebiet durch die außergewöhnliche Bergflora mit zahlreichen Endemiten aus. Die wichtigsten Arten werden kurz vorgestellt.

»Ich verlasse Teneriffa fast mit Tränen in den Augen. Ich würde am liebsten ständig hier leben«.

Alexander von Humboldt (1799)

\section{Kontinent im Kleinen}

Die Kanarischen Inseln, allen voran Teneriffa, sind ein botanischer Hotspot, der oft in einem Atemzug mit der so außergewöhnlichen Flora von Hawaii, Neuseeland oder der südafrikanischen Kapregi- on genannt wird. Teneriffa als die größte Insel im Kanarischen Archipel ist wie ein Miniaturkontinent aufgebaut. Auf relativ engem Raum bietet sie einen Querschnitt ganz unterschiedlicher Landschaftsformen. Alexander v. Humboldt, der

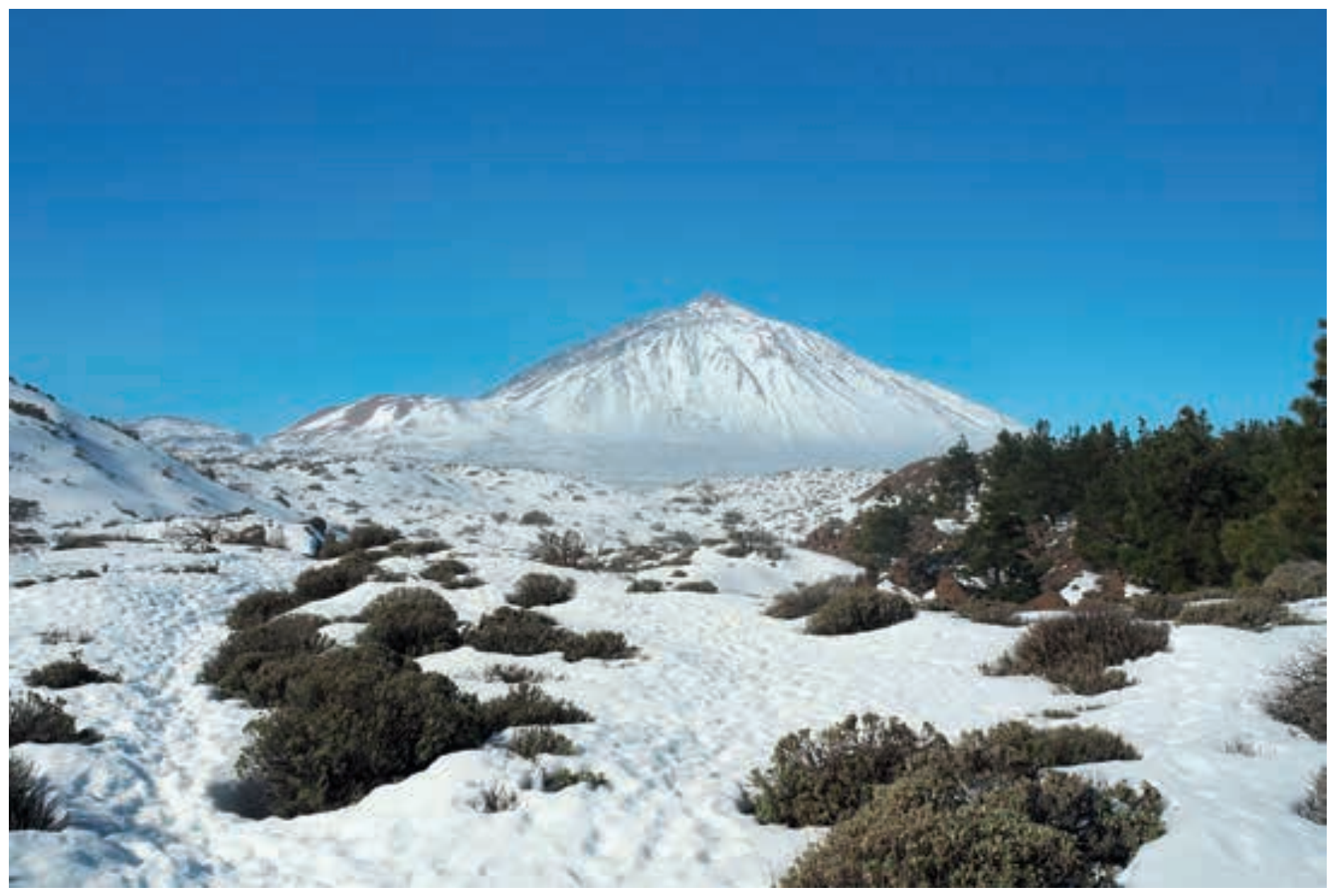

Abb. 1: Der schneebedeckte Teide im Winter. 


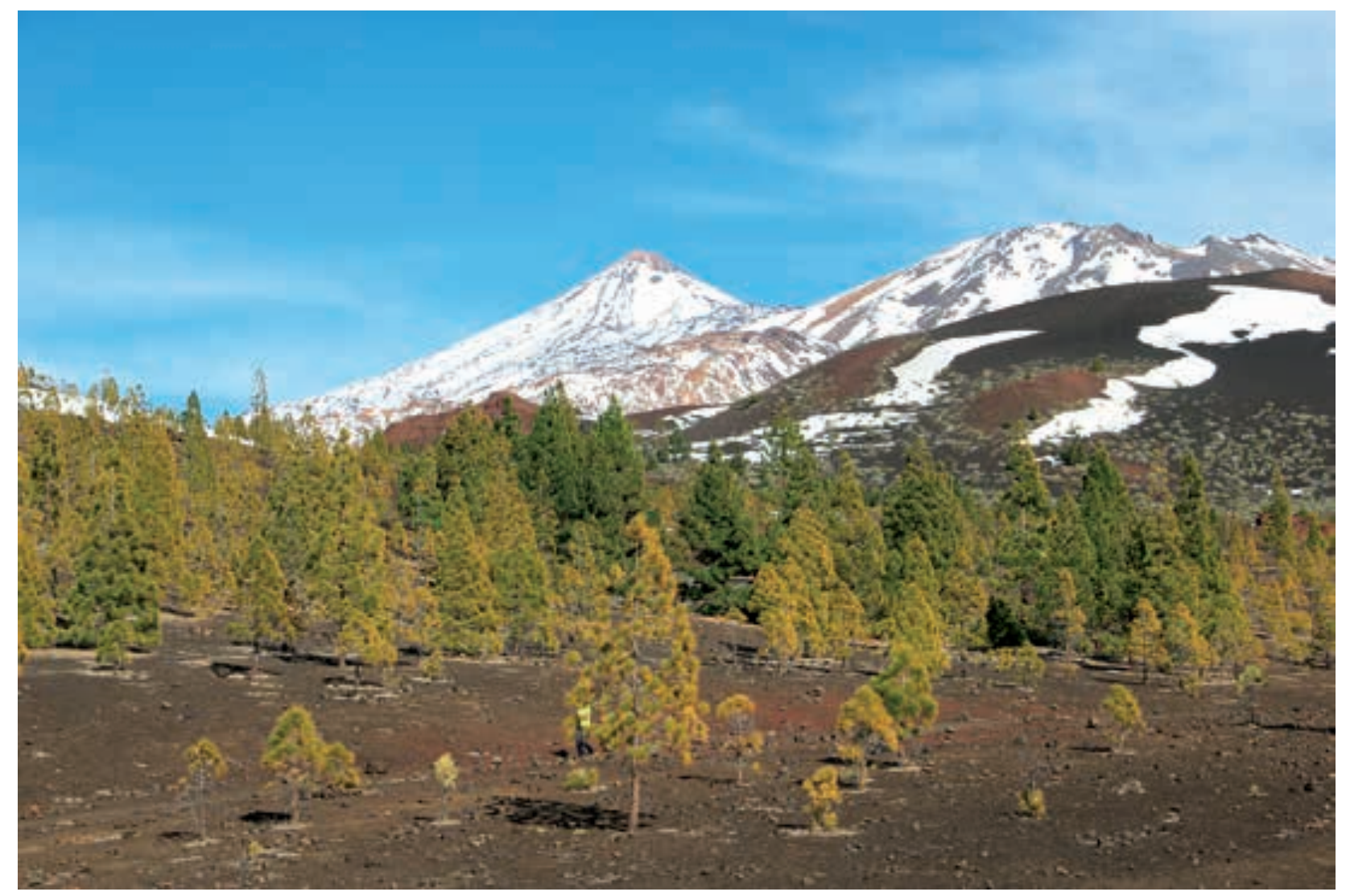

Abb. 2: Teide mit Schneeresten, davor ein lockerer Bestand der Kanaren-Kiefer (Pinus canariensis).

1799 während seiner Überfahrt nach Südamerika einen einwöchigen Aufenthalt aufTeneriffa einlegte, skizzierte mit dem Blick eines Universalgenies quasi aus dem Stehgreif heraus die Vegetationszonen der Insel, angefangen von der halbariden

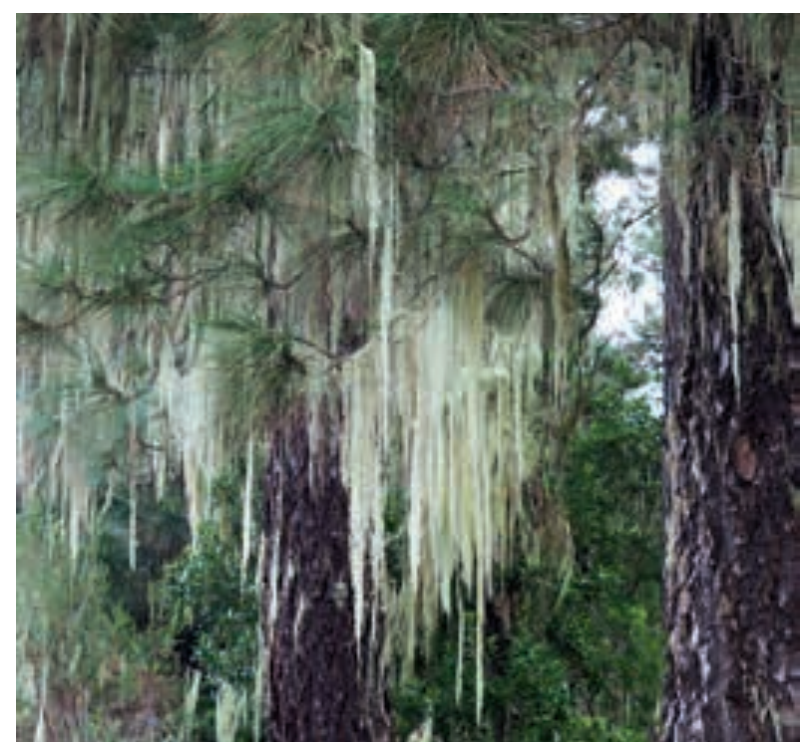

Abb. 3: Dort, wo die Passatwolke Feuchtigkeit mitbringt, die von den langen Nadel der Kanaren-Kiefern ausgekämmt wird, können sich dichte Behänge aus Bartflechten ansiedeln.
Trockenzone an der Küste bis hinauf in die subalpine Gebirgszone (Humbold 1859 ). Trotz massiver menschlicher Eingriffe in den vergangenen Jahrhunderten lässt sich das ursprüngliche Vegetationskleid auch heute noch gut erkennen. Nimmt man von Puerto de la Cruz aus die klassische Auffahrt durch das Orotava-Tal ins Gebirge, schließt sich oberhalb von Bananenkulturen und Relikten eines thermophilen Buschwaldes ein dichter subtropischer Lorbeerwald an. Im Spanischen nennt man diesen feuchten Bergwald Monteverde (Grüner Berg).

Die aus rund 20 verschiedenen Hartlaubgewächsen und einer artenreichen Begleitflora zusammengesetzte Lorbeerwaldgesellschaft verdankt ihr üppiges Grün einer aus Nordost kommenden Passatströmung, die das ganze Jahr über für konstante Feuchtigkeitszufuhr sorgt. Lorbeerwälder besetzen an den Nordhängen von Teneriffa die Höhenlagen zwischen 600 und $1200 \mathrm{~m}$. Im Unterschied zu den an der Küste so gut wie niederschlagsfreien Sommermonaten kann das Wetter hier ziemlich feucht und nebelverhangen sein. An 


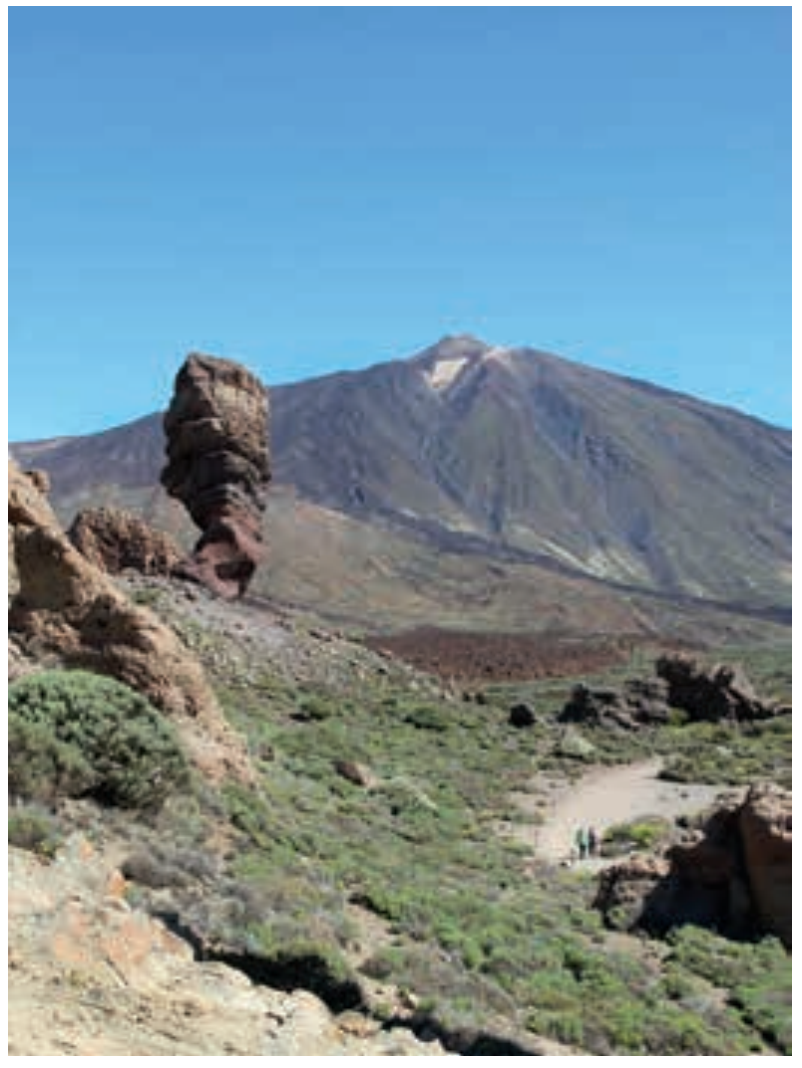

Abb. 4: Der markante und weit bekannte Roque Cinchado, dahinter der Teide.

den Rändern des Lorbeerwalds hat sich ein Baumheide-Buschwald (span. Fayal-Brezal) angesiedelt. Die Leitpflanzen dieser Zone sind Gagelbaum ( $M y$ rica faya syn. Morella faya) und Baumheide (Erica arborea), letztere wird im subtropischen Klima Teneriffas bis zu $20 \mathrm{~m}$ hoch. Die etwas trockeneren Lagen zwischen 700 und 2200 m besiedelt ein lichter Nadelwald aus endemischer Kanaren-Kiefer (Pinus canariensis). Oberhalb der Baumgrenze beginnt schließlich eine von Ginsterfluren überzogene Hochgebirgslandschaft, wie sie auf den Kanaren außer auf Teneriffa ansonsten nur auf der Nachbarinsel La Palma bestaunt werden kann.

Das Inselzentrum wird vom Pico del Teide dominiert, dem mit 3718 m höchsten Berg Spaniens. Geologen gehen davon aus, dass der Bergstock sich vor etwa 500000 Jahren infolge eines tektonischen Schubs aus dem Krater eines ursprünglich noch weitaus höheren Vulkans gebildet hat. Für die kanarischen Ureinwohner, den Guanchen, welche die Insel ab dem 2. Jahrhundert n. Chr. von Nordafrika kommend in mehreren Einwanderungsschüben bevölkerten, war der Pico

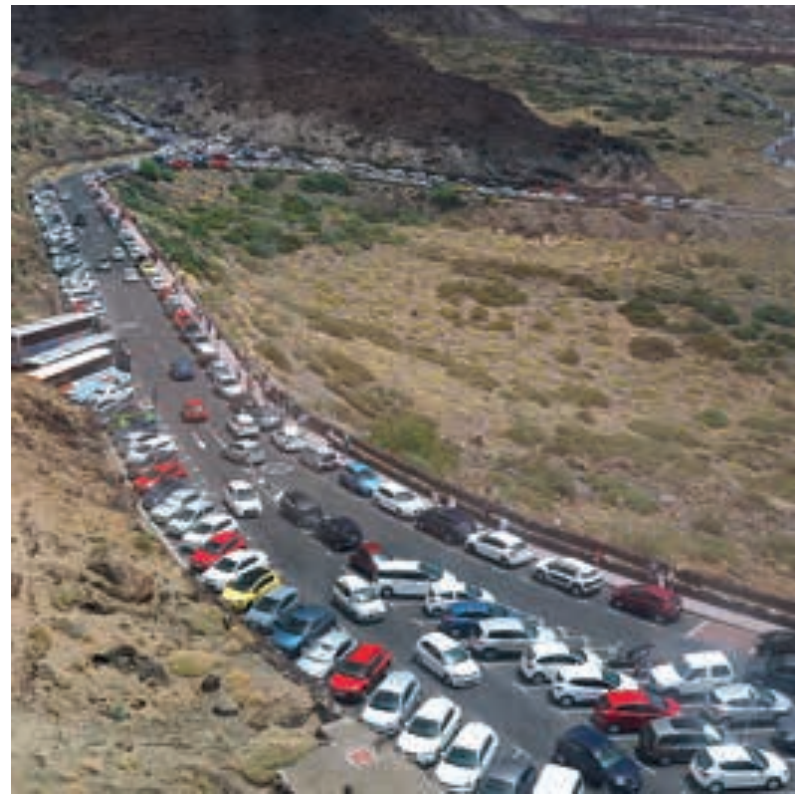

Abb. 5: Teide, Seilbahnstation und Cañadas sind von Touristen häufig sehr stark frequentiert.

del Teide eine, wie man heute sagen würde, nogo-Zone. Echeyde nannten die Guanchen den Berg, was soviel wie Hölle heißt (Ротт 2003). Der Vulkan galt als Sitz des Dämonen Guayota, der immer mal wieder unter donnerndem Getöse Feuer und Rauch ausspie und mit seinen bisweilen ins Siedlungsgebiet fließenden glühenden Lavaströmen die Einwohner in Angst und Schrecken versetzte. Die jüngste Eruption ereignete sich vor gut hundert Jahren, als am 18. November 1909 der Vulkan Chinyero, ein Nebelgipfel des Teide, die Region in Schutt und Asche legte.

\section{Der Nationalpark Teide}

Der Nationalpark mitten im geographischen Zentrum Teneriffas umfasst im Wesentlichen die sogenannten Cañadas, einen über $2000 \mathrm{~m}$ hoch gelegenen riesigen Vulkankrater mit einem Durchmesser von $17 \mathrm{~km}$, dessen Ränder bis auf $2700 \mathrm{~m}$ hinauf reichen. Im Norden des Schutzgebiets erhebt sich der namensgebende Pico del Teide. 1954 gegründet ist der Teide-Nationalpark mit knapp 19000 Hektar das viertgrößte Schutzgebiet dieser Art in Spanien. Hinsichtlich der Besucherzahlen liegt er gar an erster Stelle - jedes Jahr wollen 3 bis 4 Millionen Ausflügler und Wanderer die spektakuläre Vulkanlandschaft sehen. Damit ist zugleich eines der Hauptprobleme des Parks beim Namen genannt. Das Gebiet ist auf gut ausgebauten Straßen 


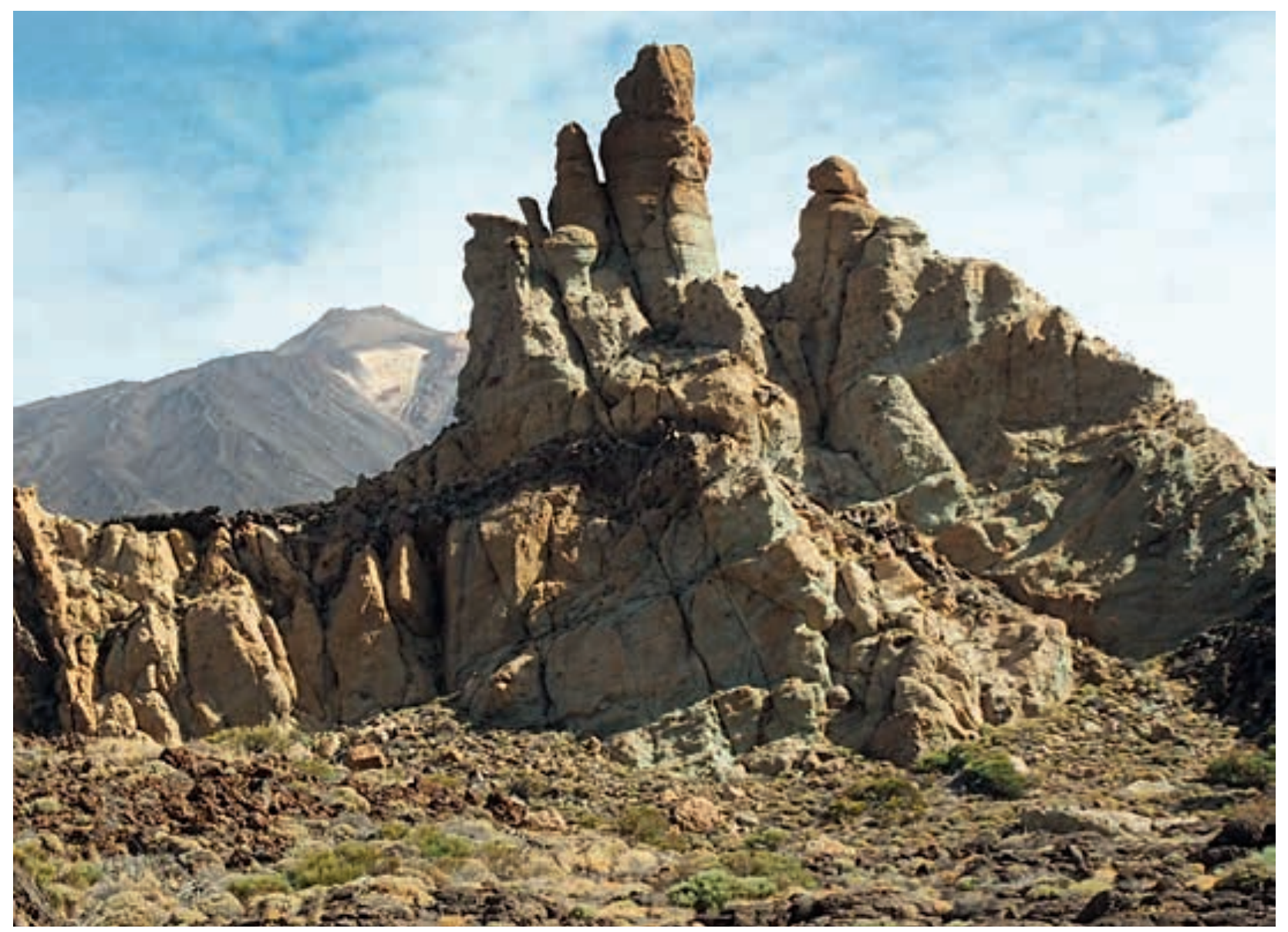

Abb. 6: Los Roques mit Teide im Hintergrund.

sehr einfach mit Auto und Bus (auch öffentlichen) erreichbar. An vielen Tagen im Jahr, vornehmlich in den Hauptferienzeiten an Ostern, im Sommer und an Weihnachten, herrscht besonders an der Zufahrt zur Teide-Seilbahn (2356 m ü. d. M.) ein Verkehrschaos fast wie in einer Millionenmetropole. Für so manchen Autofahrer braucht es da schon ein gehöriges Maß an Gelassenheit, um z.B. die am Straßenrand wuchernde Teide-Flockenblume (Cheirolophus canariensis) zu würdigen. Immer wieder werden Maßnahmen diskutiert, den bislang freien Zugang in das Schutzgebiet zu kontrollieren, sei es durch eine Eintrittsgebühr oder durch die Einführung von Shuttlebussen (beides wird beispielsweise im Nationalpark Timanfaya auf Lanzarote praktiziert). Für die Besteigung des Teide-Gipfels, die Seilbahn fährt „nur“ bis zur Rambleta auf 3555 m, gibt es schon seit Jahren eine Zugangsbeschränkung. Eine komfortable Übernachtungsmöglichkeit bietet das staatlich geführte Berghotel Parador Nacional, es liegt $2200 \mathrm{~m}$ ü. d. M.
Wie in Nationalparks üblich, ist vom Tourismus einmal abgesehen eine anderweitige wirtschaftliche Nutzung rund um den Teide nicht gestattet. Eine Ausnahme bildet lediglich die Imkerei. Während der kurzen Zeit des kanarischen Bergfrühlings produzieren die Bienen in den Cañadas einen herben Blütenhonig. Das süße Mitbringsel wird u. a. in El Sauzal in der Casa del Vino angeboten, zu der auch ein kleines Honigmuseum gehört.

\section{Weltnaturerbe Pico del Teide}

2007 nahm die UNESCO die Region in die Liste der Weltnaturerbe auf. In ihrer Begründung hob die Organisation der Vereinten Nationen einen meist nur Fachleuten bekannten Superlativ hervor: Mit rund $7500 \mathrm{~m}$ Höhe ist der Bergstock des Teide der drittgrößte Vulkan weltweit. Die $3718 \mathrm{~m}$ ü. d. M. sind sozusagen nur die „Spitze des Eisbergs“ - nochmals genauso viele Höhen- bzw. Tiefenmeter reichen bis zu seiner Basis auf dem Meeresgrund des Atlantischen Ozean hinab. Ausschlaggebend für die Ernennung zum Weltnatur- 


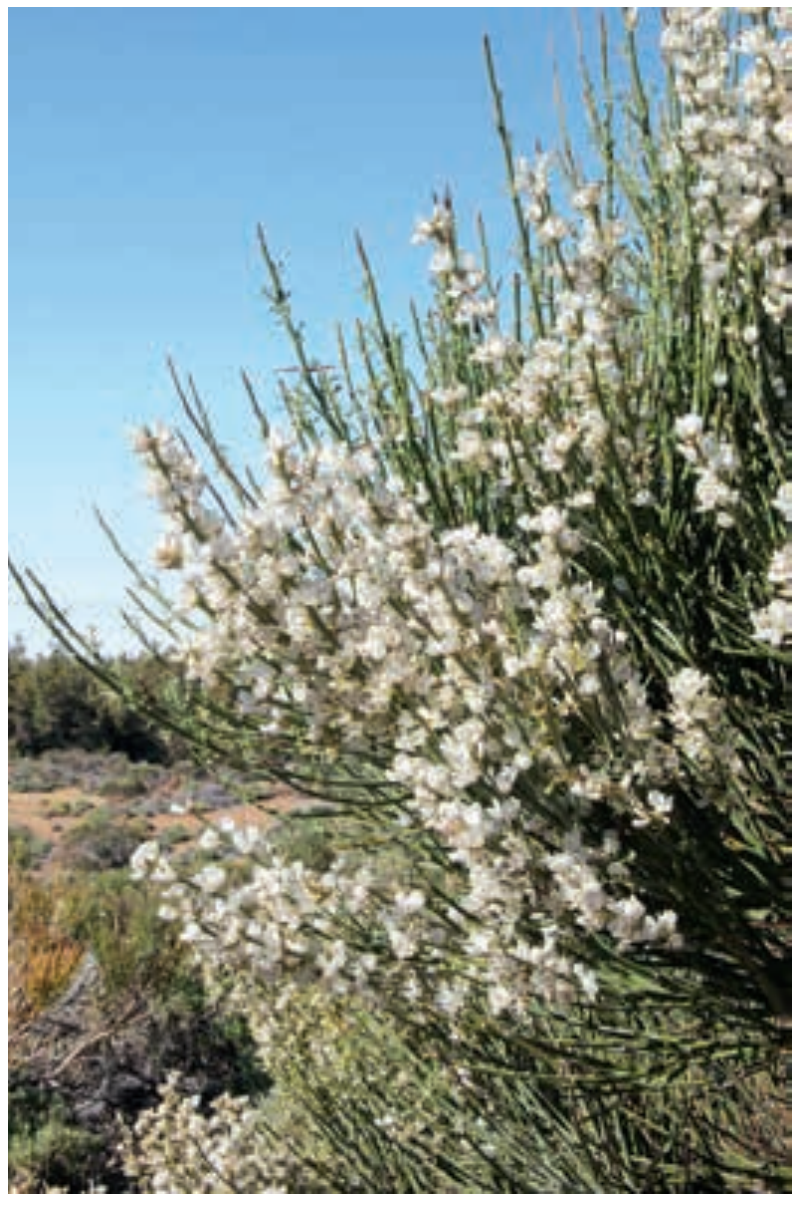

Abb. 7: Teide-Ginster (Spartocytisus supranubius).

erbe war neben der spektakulären Vulkanlandschaft nicht zuletzt die außergewöhnliche Flora. Und ebenfalls bemerkenswert: Die Atmosphäre zeichnet sich durch eine extrem klare Luft aus, was Teneriffa zu einem idealen Standort für Himmelsbeobachtungen macht. Schon 1909 gründeten Astronomen auf dem 2367 m hohen Rücken der Montaña de Izaña ein Observatorium, dem schon ein Jahr später sensationelle Fotoaufnahmen vom Halleyschen Kometen gelangen.

\section{Teide-Veilchen und andere Endemiten}

Selbst der botanische Laie ist angesichts der vielen Arten, die ausschließlich auf Teneriffa vorkommen, erstaunt. Während im Sukkulentenbusch der Küstenzone siedelnde Wolfsmilchgewächse, Aeonien und andere Endemiten sich mit teils ausgesprochen invasiven Neophyten, etwa Agave americana, Opuntia ficus-indica und dem neuerdings fast überall sich rapide ausbreitenden Federborstengras (Pennisetum setaceum), den Platz an der Sonne teilen müssen, blieb die Kanarenflora im

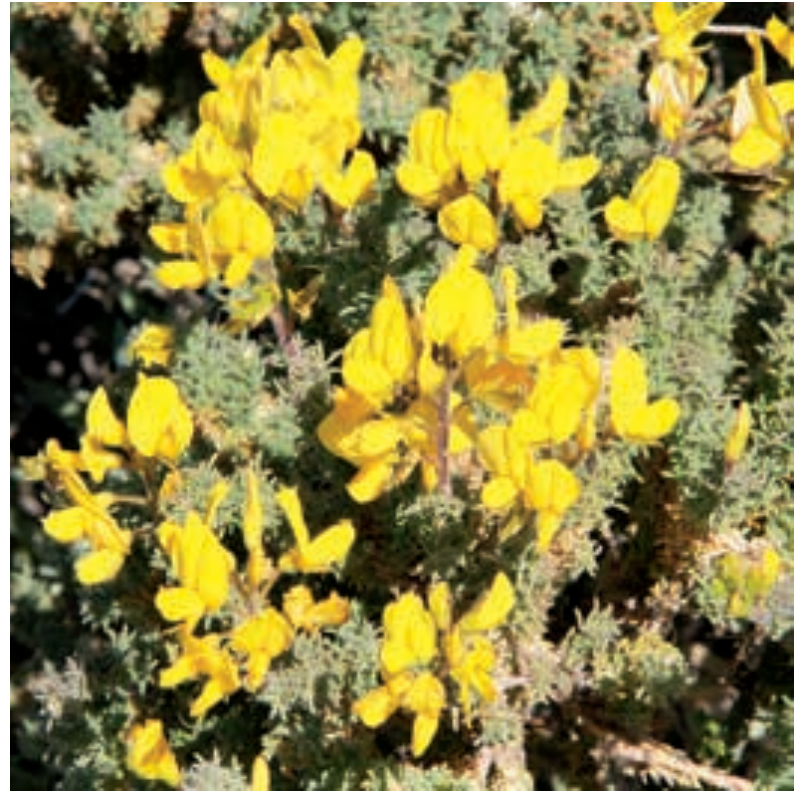

Abb. 8. Klebrige Drüsenfrucht (Adenocarpus viscosus ssp. viscosus).

Gebirge bislang weitgehend von Fremdeinflüssen verschont. Angesichts der Höhenlage, intensiver Sonneneinstrahlung und den für das ansonsten ausgeglichene kanarische Klima ungewöhnlich hohen tageszeitlichen Temperaturschwankungen von mitunter bis zu fast $20 \mathrm{Grad}$ kann sich im Nationalparkgebiet nur eine hoch spezialisierte Gebirgsflora behaupten. Sie muss zudem mit relativ geringen Niederschlägen von unter $500 \mathrm{~mm}$ im Jahresmittel auskommen (Llaría López 2001). Ungeachtet der subtropischen Breite kann es in höheren Lagen im Winter mitunter schneien, während zur gleichen Zeit an der Küste sonnig-warmes Badewetter herrscht.

Umso erstaunlicher präsentiert sich die Flora. Im Gebiet des Teide-Nationalparks kommen rund 200 Arten von Blütenpflanzen vor. Davon sind 58 Kanarenendemiten und immerhin 12 sind ausschließlich im Nationalpark anzutreffen - angesichts der relativ kleinen Fläche eine durchaus beachtliche Quote. Zu diesen gehören etwa Klebrige Drüsenfrucht (Adenocarpus viscosus ssp. viscosus), Cañadas-Kanarenmargerite (Argyranthemum tenerifae), Aubers Natternkopf (Echium auberianum) und nicht zuletzt WiLDPRETs Natternkopf (E. wildpretii). Das Teide-Veilchen (Viola cheiranthifolia Нumb. \& Bonpl.), eines der großen Stars der Kanarenflora, wird man allerdings kaum zu 


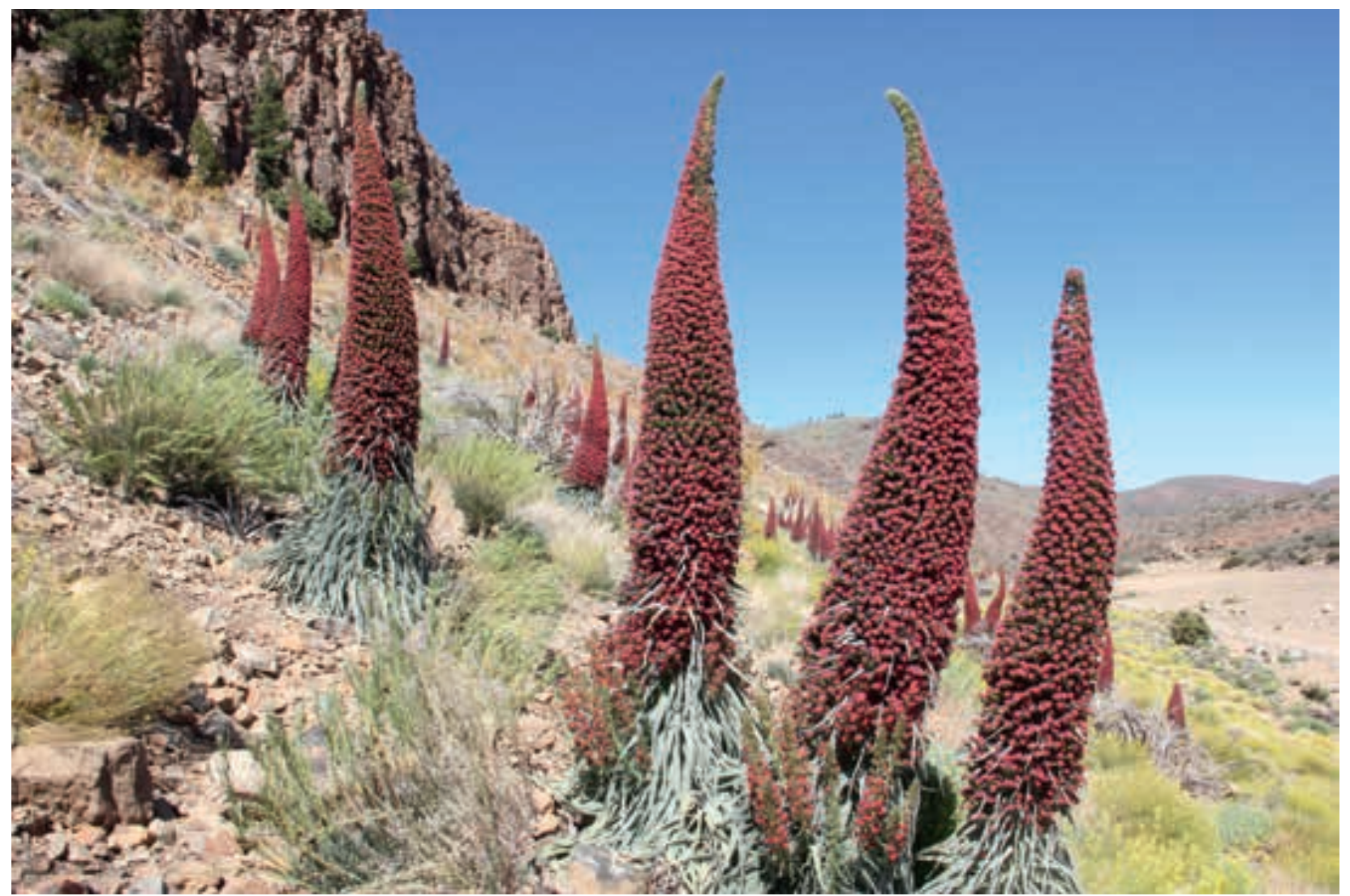

Abb. 9: Wildprets Natternkopf (Echium wildpretii).

Gesicht bekommen. Nicht nur weil es sehr selten ist, sondern weil es ausschließlich auf unwirtlichen Geröllhängen in Lagen zwischen 2600 und $3400 \mathrm{~m}$ siedelt. Außer am Teide kommt es in freier Natur lediglich an der benachbarten Montaña Blanca und der Montańa Guajara vor. Humboldt entdeckte die hell- bis dunkelvioletten Blüten während seiner Teide-Besteigung auf $3390 \mathrm{~m}$ Höhe (Нumbold т 1859). Noch seltener ist das Teide-Edelweiß (Gnaphalium teydeum), es ist ausschließlich auf die Gipfelzone des Teide begrenzt (Schönfelder \& Schönfelder 2012).

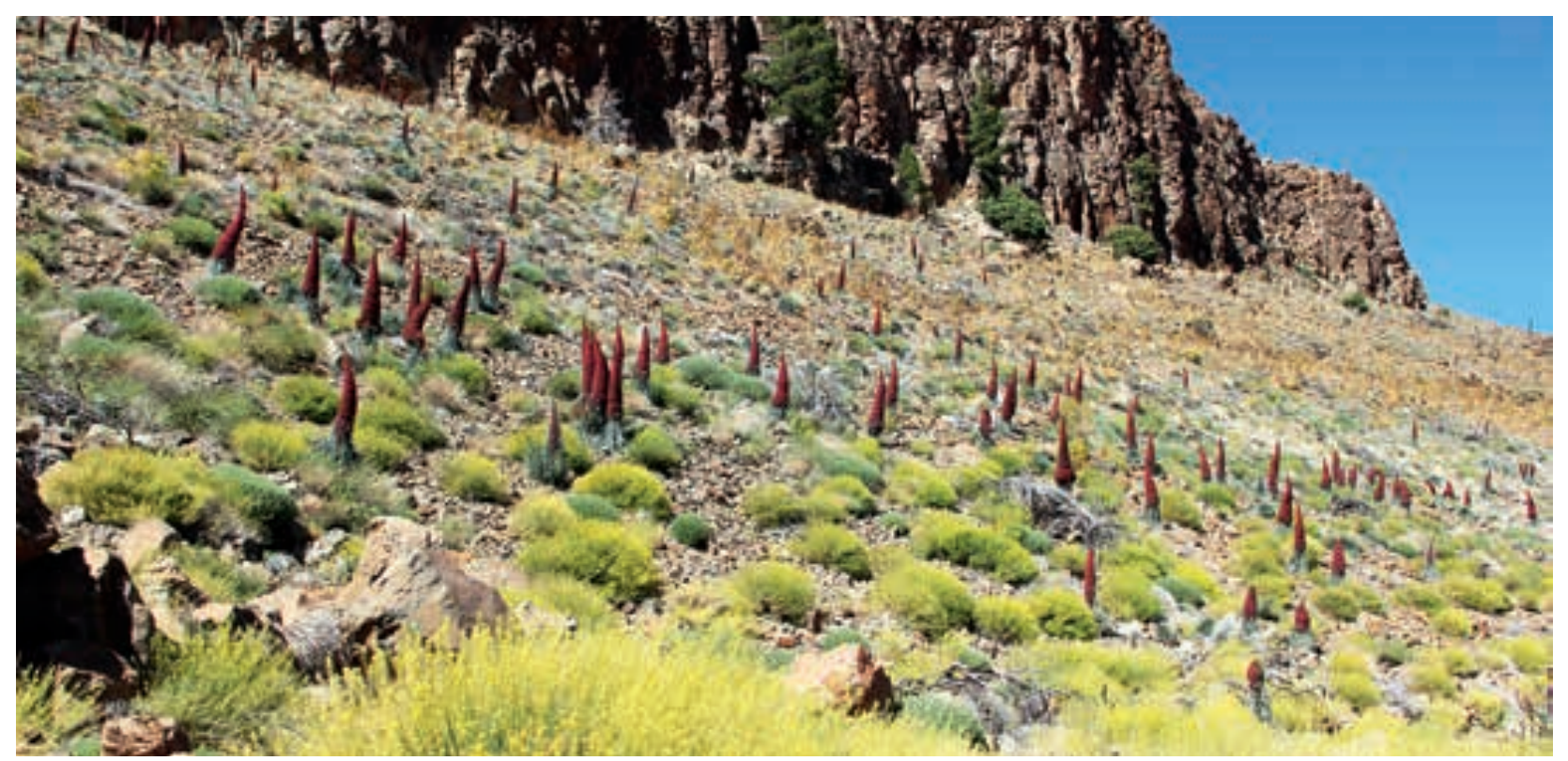

Abb. 10: Ein beeindruckender, individuenreicher Bestand von WILDPRETs Natternkopf an der Fortaleza. 


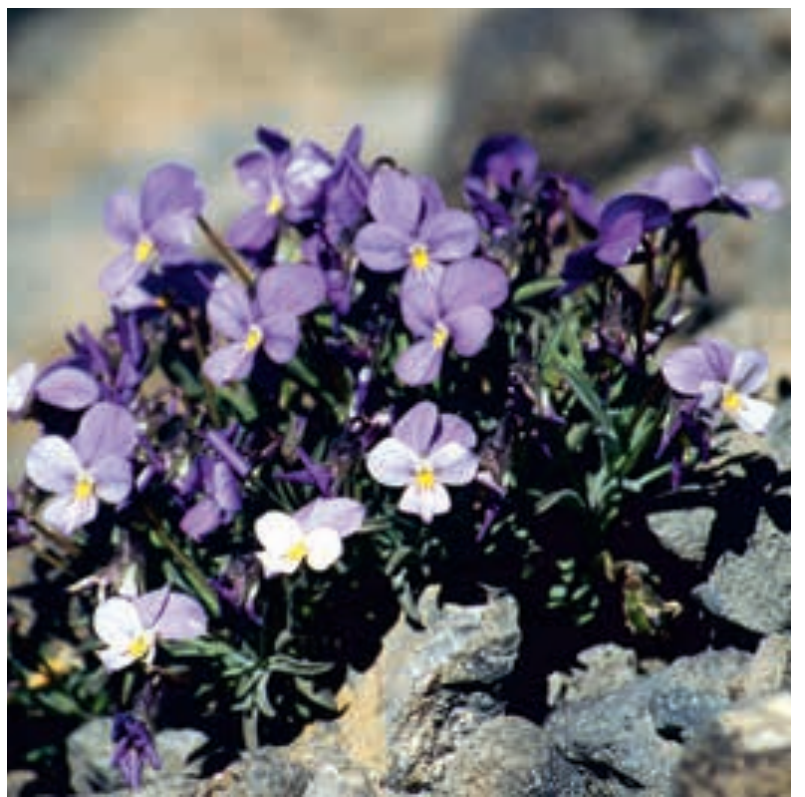

Abb. 11: Teide-Veilchen (Viola cheiranthifolia).

\section{Ginsterfluren am Fuß der Vulkanschlote Los Roques}

Der Bergfrühling im Nationalpark beginnt Ende März, wenn auf 2000 m Höhe der Besen-Schöterich (Erysimum scoparium) seine weiß-violetten Kronblätter öffnet. Der auch Teide-Lack genannte Halbstrauch ist mit dem in Mitteleuropa verbreiteten Goldlack (E. cheiri) verwandt. Richtig los mit dem Frühling geht es jedoch erst im Mai. Dann verwandelt der Teide-Ginster (Spartocytisus supranubius) die Cañadas in ein weißes Blütenmeer, dazwischen setzen die rosafarbenen Blütenköpfe des Behaarten Federkopf (Pterocephalus lasiospermus) markante Farbtupfer. Wie es der Name supranubius erklärt, ist die Ginsterart ausschließlich über den Wolken zuhause. An geschützten Stellen klettern die Sträucher am Südhang des Teide bis in Lagen von $3200 \mathrm{~m}$ hinauf. Doch so hoch muss der Besucher nicht steigen, ausgedehnte Bestände gibt es etwa in der Ucanca-Ebene auf $1900 \mathrm{~m}$ und etwas oberhalb davon an den freigewitterten Vulkanschloten Los Roques. Mit dem berühmten Roque Cinchado, dem geologischen Wahrzeichen Teneriffas, sind die bizarren Felsformationen der Hauptanlaufpunkt im Nationalpark. Nahebei kann man auf der Außenterrasse der Cafeteria des Berghotels Parador Nacional verweilen und das einzigartige Panorama auf den Pico del Teide genießen.

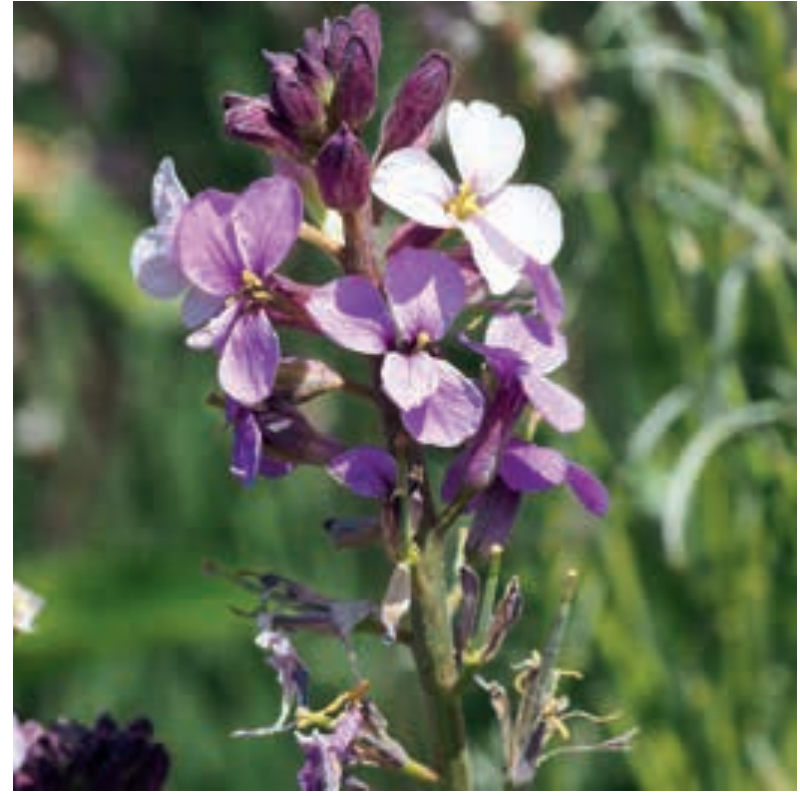

Abb. 12: Besen-Schöterich (Erysimum scoparium).

\section{Exkursion zu den Riesennatternköpfen an der Fortaleza}

Im Osten des Nationalparks macht im Besucherzentrum von El Portillo (2050 m ü. d. M.) eine Dauerausstellung mit der Flora, Fauna und der geologischen Entstehung der Teide-Region bekannt. In dem zugehörigen kleinen Botanischen Garten ist mehr als die Hälfte der im Nationalpark vorkommenden Arten präsent. El Portillo ist zugleich ein hervorragender Ausgangspunkt für Wanderungen durch die Cañadas. Da es meist nur leicht auf und ab geht, eignet sich das von der Nationalparkverwaltung ausgeschilderte Wege-

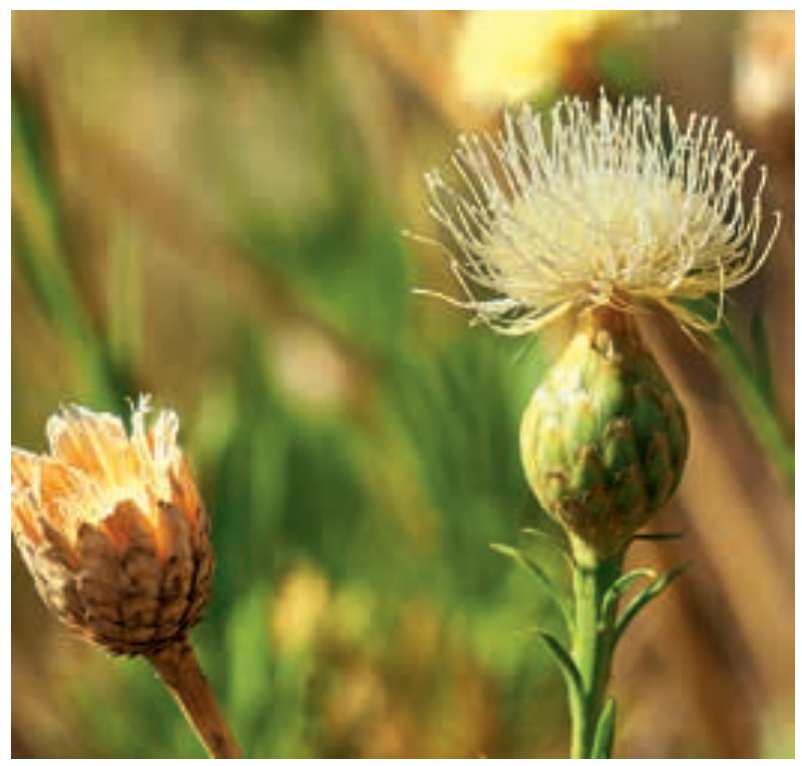

Abb. 13: Teide-Flockenblume (Cheirolophus canariensis). 


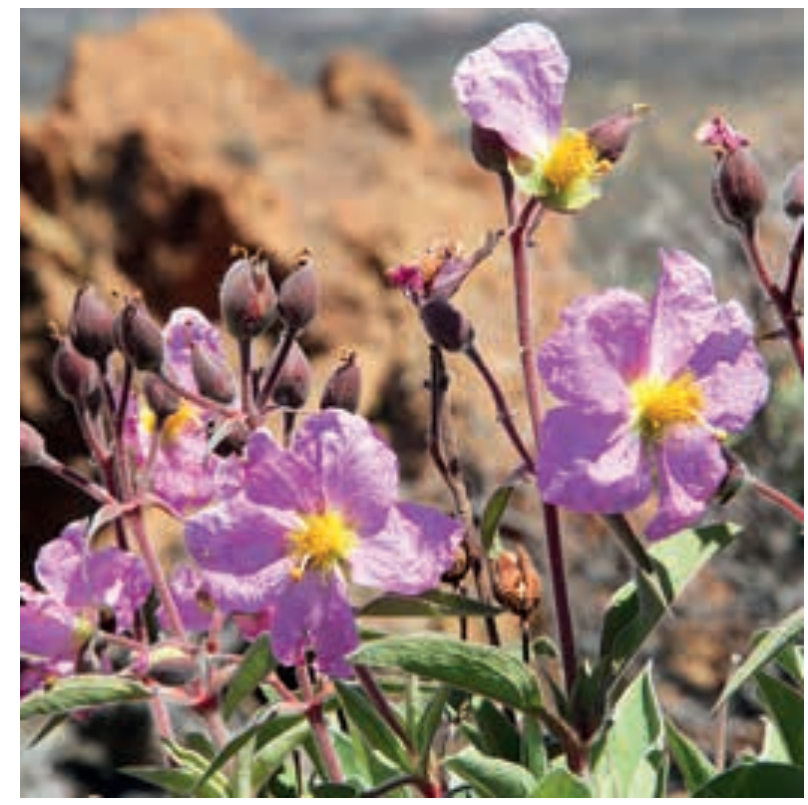

Abb. 14: Osbeckienblättrige Zistrose (Cistus osbeckiifolius).

netz auch für weniger konditionsstarke Wanderer (Goetz 2017a, b). Aus botanischer Sicht die Attraktion schlechthin ist die hin und zurück etwa dreistündige Tour zur Fortaleza, einem mächtigen Felsriegel, der als Teil einer ursprünglichen Kraterwand die Nordgrenze des Nationalparks bildet. Zur richtigen Zeit am richtigen Ort kann man am Fuß der schroff abfallenden Südwand den größten Bestand von Wildp RETs Natternkopf (Echium wildpretii) bestaunen. Die Blütezeit der bis zu $3 \mathrm{~m}$ hohen unverzweigten Pflanze reicht von Ende Mai bis Ende Juni. Den prächtigen Blütenstand besetzen Tausende von leuchtend roten Trichterblüten. Das Raublattgewächs ist mit der Teide-Rauke (Descurainia bourgeauana) vergesellschaft, deren senfgelbe Blüten einen attraktiven Kontrast zu den roten Blütenkerzen setzen. Von den Einwohnern Teneriffas wird der Riesennatternkopf gemeinhin Stolz von Teneriffa genannt. Besser lässt sich die Wertschätzung dieser außergewöhnlichen Pflanze kaum ausdrücken.

Vom Reich der Natternköpfe kann man auf einem sandigen Pfad in wenigen Minuten zur Degollada del Cedro, dem Zedernsattel, aufsteigen. Von dort wusste der Schweizer Botaniker HerMANN CHrist (1886) auf seiner „Frühlingsfahrt“ zu berichten, dass eine stolze Gruppe Zedern-Wacholder (Juniperus cedrus) dem Wanderer Schatten spendet; heute stehen dort zwei große Kanarenkie-

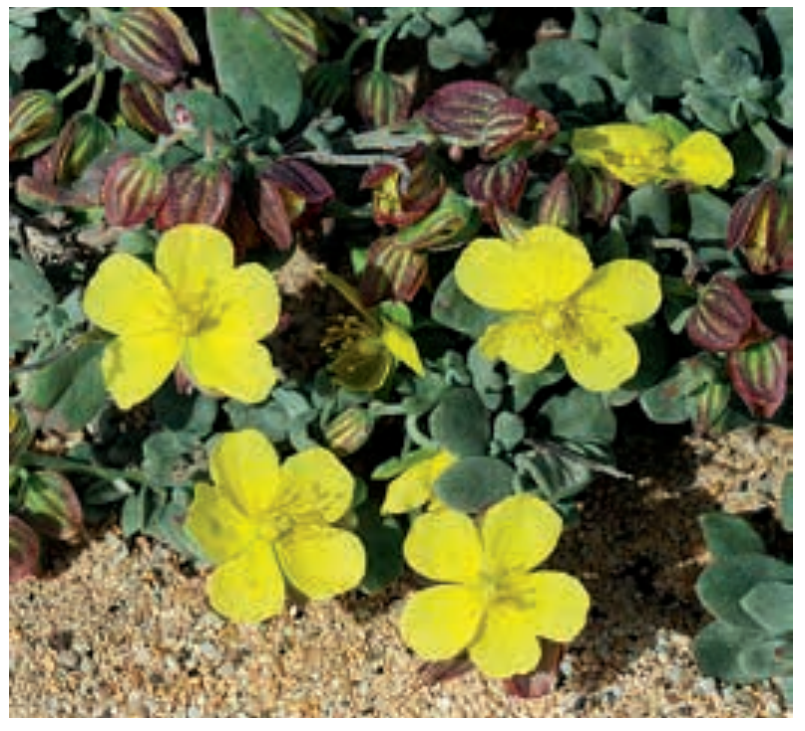

Abb. 15: Kanarisches Sonnenröschen (Helianthemum canariensis); gut zu erkennen die verschieden gefärbten Aschestückchen im Boden.

fern neben einer pittoresken Kapelle. Vom Zedernsattel ist es nicht mehr weit auf den Rücken der Fortaleza (2150 m ü. d. M.). Neben dem wie Fenchel aussehenden Links Rutenkraut (Ferula linkii) trifft man dort unverhofft auf die im Frühsommer rosa blühende Osbeckienblättrige Zistrose (Cistus osbeckiifolius). Sie ist eine von sieben auf den Kanaren beheimateten Zistrosengewächse, doch im Unterschied etwa zur Montepellier-Zistrose $(C$. monspeliensis) und der Beinwellblättrigen Zistrose (C. symphytifolius) sieht man sie extrem selten.

In der Region El Portillo ist auch das BergSonnenröschen (Helianthemum juliae) zuhause. Dieser im Spanischen Jarilla de Cumbre genannte Nationalpark-Endemit wurde erst 1986 von Wolfredo Wildpret de la Torre beschrieben, einem Enkel des Schweizer Gärtners Hermann WILDPRET, nach dem der oben erwähnte Natternkopf benannt ist (vgl. Steinecke 2017). Das Berg-Sonnenröschen siedelt ausschließlich in Lagen zwischen 2000 und 2300 m. Wie andere auf den Kanaren vorkommende Verwandte macht es durch seine gelben Blüten auf sich aufmerksam. Anders als das auch auf den Nachbarinseln anzutreffende Kanaren-Sonnenröschen (H. canariensis), das niedere Lagen bevorzugt und kaum über eine Wuchshöhe von 0,25 m hinauskommt, kann das Berg-Sonnenröschen mehr als doppelt so hoch werden. Um den Fortbestand dieser seltenen 


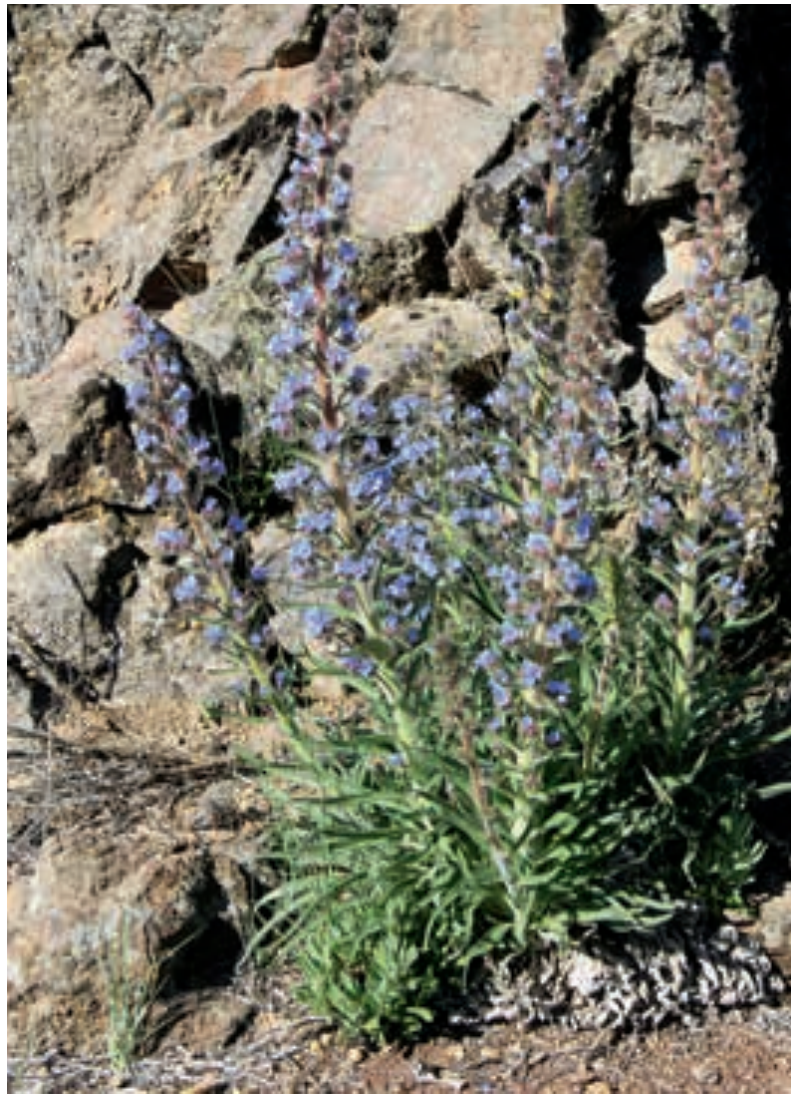

Abb. 16: Aubers Natternkopf (Echium auberianum).

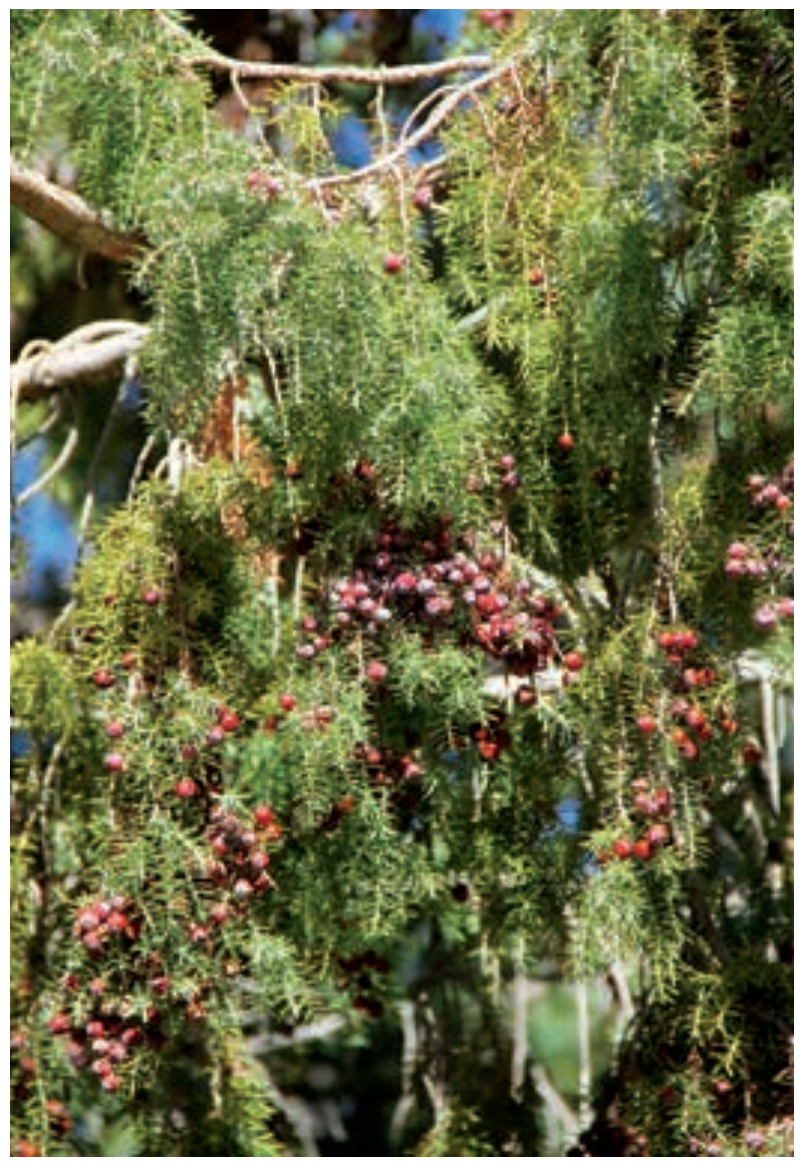

Abb. 17: Zedern-Wacholder (Juniperus cedrus).
Art zu sichern, führte die Nationalparkverwaltung in den vergangenen Jahren mehrere erfolgreiche Pflanzaktionen durch, wodurch sich die Population spürbar erholt hat und sich von ursprünglich einigen wenigen hundert Individuen auf etwa 4000 erhöht hat.

Wer die eindrucksvolle Landschaft am Teide und dessen Pflanzenwelt erkunden möchte, sollte sich auf keinen Fall entgehen lassen, gegebenenfalls von der Bergstation der Seilbahn bis auf den Gipfel des Vulkans zu steigen. Hierfür benötigt man eine Genehmigung. Unter der unten angegebenen Internetseite können sich Wanderer online eine kostenlose Genehmigung zur Besteigung des Teide ausstellen lassen Diese sollte mindestens zwei Monate im Voraus beantragt werden, denn der Andrang auf den höchsten Gipfel Spaniens ist riesig und pro Zeitfenster wird nur eine begrenzte Personenanzahl zugelassen.

\section{Literatur}

BRAMWELl, D. 2014: Flora der Kanarischen Inseln. - Madrid. Christ, H. 2016: Eine Frühlingsfahrt nach den Kanarischen Inseln. - Norderstedt (unveränderter Nachdruck von 1886).

Goetz, R. 2017a: Flora der Kanarischen Inseln. - München. Goetz, R. 2017b: Kanarische Inseln - Botanische Wanderungen. - München.

Humboldt, A. von 1859-1861: Reise in die Aequinoctial-Gegenden des neuen Continents. - Stuttgart.

Llaría lopez, M. A., Gómez, J. A. R. \& Hernández Álvarez, J. C. 2001: Der Teide-Nationalpark Besuchsführer. - Islas Canarias.

Pott, R., Hüppe, J. \& Wildpret de la Torre, W. 2003: Die Kanarischen Inseln - Natur- und Kulturlandschaften. Stuttgart.

Schönfelder, I. \& Schönfelder, P. 2012: Die Kosmos-Kanarenflora. - Stuttgart.

Steinecke, H. \& Dörken, V. M. 2017: Der botanische Garten von Orotava auf Teneriffa. - Palmengarten 81/1: 12-18.

\section{Internetseiten}

www.floradecanarias.com

gibt in Wort (in spanischer Sprache) und Bild einen Überblick über die Flora der Kanarischen Inseln

www.volcanteide.com

informiert über Betriebszeiten und Preise der Teide-Seilbahn (Teleférico del Teide)

www.reservasparquesnacionales.es 